\author{
Marija BRADAS̆ \\ Università Ca' Foscari Venezia
}

\title{
APPUNTI SULLA PROSA SAGGISTICA DI PETAR KASANDRIĆ
}

Parole chiave: Petar Kasandrić, traduzioni, giornalismo, prosa saggistica.

Oggi Petar Kasandrić (1857-1926) si ricorda come giornalista, critico letterario e traduttore. Tuttavia la sua attività di mediatore culturale, lunga e poliedrica, non è stata messa adeguatamente in luce: se viene spesso nominato in modo incidentale, pochi sono i lavori dedicati interamente ad aspetti delle sue opere, e manca un testo che passi dettagliatamente in rassegna la sua varia e ricca produzione. ${ }^{1}$

Nato sull'isola di Hvar (Lesina), ${ }^{2}$ Kasandrić compì gli studi liceali a Dubrovnik (Ragusa). Nel 1887 divenne impiegato contabile presso il Namjesništvo Dalmacije (Governatorato della Dalmazia) a Zara, allora capitale della Dalmazia; alla fine dell'anno gli fu affidata la redazione di

*marija.bradas@unive.it

${ }^{1}$ Da una lettera all'amico Ante Petravić emerge la delusione di Kasandrić per la poca attenzione che i suoi lavori suscitavano presso i contemporanei: «Lei mi ha coperto di lodi, che davvero non merito, ma che hanno certamente e in grande misura compensato la noncuranza con la quale il nostro pubblico ha incontrato il mio coscienzioso, anche se davvero limitato, lavoro nel campo della nostra storia letteraria». Cfr. H. Morović, Iz prepiske Petra Kasandrića sa A. Petravićem, «Zadarska revija» 5, 1970, pp. 523-538: 526-527, salvo avviso in contrario, le traduzioni sono di chi scrive.

${ }^{2}$ I dati sulla vita di Kasandrić si riscontrano in vari dizionari biografici croati, serbi e jugoslavi (cfr. Hrvatski biografski rječnik, Srpski biografski rečnik, Leksikon pisaca Jugoslavije), ma informazioni utili si trovano anche in V. MAšrrović, «Smotra dalmatinska» i Petar Kasandrić, «Jadranski zbornik», III/1958, Rijeka-Pula, pp. 371-384, che si basa sui dati forniti dalla figlia di Kasandrić, nonché nel saggio di Nikša Stipčević, pubblicato come prefazione alla traduzione di Gorski vijenac, e adesso in N. STIPČEvić, Poređenja, Beograd, Zavod za udžbenike i nastavna sredstva, pp. 85-113. 
«Smotra dalmatinska», organo ufficiale del governo con cui il Namjesništvo voleva partecipare al fervido dibattito giornalistico dell'epoca, come nota Vjekoslav Maštrović. ${ }^{3}$ Kasandrić ne fu redattore dal primo numero del 1888 all'ultimo del 1918, trent'anni durante i quali curò la redazione anche dell' «Objavitelj dalmatinski» (1894-1918) e di «Glasnik Matice dalmatinske» (1903-1904). La «Smotra dalmatinska» pubblicava articoli di vario argomento, dalla politica all' economia, dal commercio alle navigazioni, e comprendeva anche interventi di carattere critico-letterario in una sezione dedicata ad autori come Andrija Kačić Miošić, Vuk Stefanović Karadžić, Johann Wolfgang Goethe, Niccolò Tommaseo, Prosper Mérimée. ${ }^{4}$

Dalla rassegna degli interventi d'interesse letterario pubblicati su queste e altre riviste zaratine emerge l'interesse di Kasandrić per Kačić Miošić e il suo Razgovor ugodni, tema su cui torna in varie sedi e lungo tutta la sua carriera di giornalista e di critico. Particolarmente interessanti sono anche gli interventi dedicati alla poesia popolare, apparsi sull' "Annuario dalmatico» e sull' «Iskra», ma anche i lavori su Marko Marulić e Hanibal Lucić. Spesso Kasandrić firmava i suoi contributi apparsi sull' «Iskra» con la sola lettera $\Phi$ dell'alfabeto greco (e cirillico), un'usanza le cui ragioni non sono chiare nemmeno a Vjekoslav Maštrović, ma che non può non ricordare le tre lettere straniere KXY con cui Tommaseo firmava gli articoli sull' «Antologia» di Vieusseux. Capitava pure che Kasandrić non firmasse affatto i propri articoli, soprattutto quelli d'occasione: un anonimato che creerà non pochi problemi ai curatori della sua bibliografia complessiva.

Kasandrić scriveva sia in italiano che in serbo-croato con la stessa facilità e ricercatezza di stile, doti ammirate anche dai suoi contemporanei. «Rispetto a tutta la generazione di giornalisti e scrittori del suo tempo, Kasandrić scrisse nel più perfetto italiano», avrebbe affermato Ante Petravić..$^{5}$ Marko Car lo definiva «un letterato colto che con egual abilità padroneggia sia la lingua serba che quella italiana $» .{ }^{6}$ Dimostra infatti la sua conoscenza della lingua e del verso italiano esordendo nella vita culturale dalmata con la raccolta in italiano Prime liriche e ultime, uscita a Zara nel 1879. ${ }^{7}$ Annunciando lo sbocciare di una poesia, il titolo annuncia nello stesso tempo la determinazione dell'autore - un giovane ventiduenne - di non scrivere più versi. In un saggio dedicato alla breve esperienza poetica

\footnotetext{
${ }^{3}$ V. Maštrović, «Smotra dalmatinska» i Petar Kasandrić, cit., pp. 371-72.

${ }^{4}$ Ivi, pp. 376-77.

${ }^{5}$ Ivi, p. 382.

${ }^{6}$ M. CAR, Pietro Kasandrić, Canti popolari serbi e croati, «Letopis Matice srpske» 294/4, 1913, pp. 90-95: 90.

${ }^{7}$ P. CAssandrich, Prime liriche e ultime, Zara, Tipografia Vitaliani e Janković, 1879.
} 
di Kasandrić, Mario Festini spiega questa decisione con la consapevolezza dell'autore di non avere le doti di poeta, nonostante la profonda conoscenza delle leggi metriche e del lessico poetico italiano. ${ }^{8}$ I testi poetici di Kasandrić, in cui si possono notare influenze della poesia del primo Ottocento italiano, preannunciano alcune caratteristiche della prosa saggistica degli anni successivi. Vi si riscontrano strutture ternarie («Alta è la notte, placida, serena», «lento, uggioso, tranquillo», «mesta, silente, pensierosa», «lenta, assidua, uggiosa», «miti, tiepidi, olenti»), che si basano principalmente sul rapporto di sinonimia tra aggettivi, e più raramente strutture binarie («mesta e serena», «e triste e inaridita», «rosea salute e candida allegria»).

Abbandonata l'impresa di una poesia propria, Kasandrić incanala i suoi interessi nel lavoro di traduttore di testi letterari, impegnandosi principalmente in traduzioni di poesia popolare serba e croata, che vengono di frequente poste allo stesso livello della raccolta illirica di Tommaseo, notoriamente la traduzione meglio riuscita e più fortunata nella cultura italiana. Kasandrić è indubbiamente il traduttore dei canti popolari serbi e croati che ha dedicato più tempo ed energie al suo impegno. Le traduzioni ebbero quattro edizioni diverse: ${ }^{9}$ non erano mere ristampe delle edizioni precedenti, ma piuttosto la dimostrazione di un impegno duraturo e di una dedizione non comune. Inoltre, Kasandrić è l'unico traduttore che si possa definire perfettamente bilingue, e non meno importante per la riuscita del suo lavoro è stata la sua doppia «coscienza letteraria», intrisa della secolare tradizione popolare italiana, e insieme della letteratura serba e croata, colta e popolare, di cui era uno studioso stimato.

Kasandrić continuò a occuparsi di poesia popolare durante tutto il suo periodo zaratino, ma anche dopo il 1919, quando si trasferì a Roma, dove gli fu assegnato l'incarico di addetto all'ufficio stampa presso l'ambasciata del Regno dei serbi, croati e sloveni, posizione che mantenne fino alla morte nel 1926. A questo periodo risalgono la sua traduzione di Gorski vijenac di Petar II Petrović Njegoš, molto lodata dalla critica, ${ }^{10}$ nonché la collaborazione con

${ }^{8}$ M. Festini, Le prime liriche e ultime di Petar Kasandrić, «Radovi. Razdio filoloških znanosti», 21/12, 1981/82, pp. 251-264.

${ }^{9}$ P. CASSANDrich, Canti popolari epici serbi; versione metrica, Zara, Sant'Artale, 1884; ID., Canti popolari serbi e croati, Venezia, Veneto Arti Grafiche, 1913; ID., Canti popolari serbi e croati, Milano, Fratelli Treves, 1914; ID., Canti popolari serbi e croati, Lanciano, Carabba, 1923.

${ }^{10}$ Completata poco prima della morte, la traduzione di Gorski vijenac è pubblicata postuma a Belgrado nel 1999, grazie all'impegno di Nikša Stipčević: Petra Kasandrića prevod "Gorskog vijenca” Petra II Petrovića Njegoša, SANU, Beograd, 1999. La traduzione ha avuto un'altra edizione belgradese a cura di Nikola Damjanović con contributi di Nikša Stipčević, Mirka Zogović e Jovan Deretić: Petar II Petrović NJegoš, 
la rivista «Europa orientale». Di particolare importanza è il suo saggio su Njegoš, ${ }^{11}$ un'altra dimostrazione che il lavoro di traduttore andava spesso di pari passo con quello di critico letterario, il cui obiettivo era parlare al pubblico italiano «dell'animo e dell'ingegno di questo principe balcanico». La stessa dittologia «dell'animo e dell'ingegno» conta un'altra ricorrenza nel saggio e richiama alla memoria il lavoro giovanile di Tommaseo dedicato all'amico dalmata Antonio Marinovich, intitolato Dell'animo e dell'ingegno di Antonio Marinovich. ${ }^{12}$

Impegnato in una missione di mediazione culturale su entrambe le sponde dell'Adriatico, Kasandrić pubblica articoli sulla letteratura serba e croata in Italia e, intanto, continua a collaborare con le riviste serbe e croate inviando contributi di argomento italiano. Nel 1924 esce un articolo dedicato a Tommaseo in occasione del cinquantesimo anniversario della morte. L'articolo, diviso in due parti e uscito sulla rivista belgradese «Novi život», era pensato come una recensione al carteggio tra Tommaseo e Capponi, mirata in particolare sulle questioni slave trattate nell'epistolario. Tuttavia vi si leggono osservazioni di respiro molto più ampio che abbracciano tutta l'opera di Tommaseo riguardante il mondo slavo. Kasandrić riconosce in questo carteggio, come farà anche Contini, ${ }^{13}$ la massima espressione dell'umanità di Tommaseo e si sofferma in particolar modo sulle difficoltà che il poeta viveva per la sua doppia anima slavo-italiana, criticando inoltre le manipolazioni politiche della personalità di Tommaseo compiute in Dalmazia sia da parte italiana che da parte slava. Con questa presa di posizione, da osservatore esterno e indipendente, Kasandrić in un certo modo precede il lavoro di Mate Zorić e Nikša Stipčević. Il suo impegno non fu influenzato da visioni politiche o estetiche aprioristiche. Dalle sue osservazioni non traspare l'intenzione di provare la supremazia di una produzione letteraria sull'altra, né vi si notano ideologie politiche. Bisogna tuttavia condividere la posizione di Stipčević che colloca l'impegno di Kasandrić in quell'atmosfera dalmata di fine Ottocento in cui alla cittadinanza italiana si voleva dimostrare che

Ghirlanda della montagna, traduzione di Petar Kasandrić, Beograd, Njegoševa zadužbina, 2003, nonché un'edizione montenegrina: Gorski vijenac Petra II Petrovića Njegoša u italijanskom prevodu Petra Kasandrića, a cura di Vesna Kilibarda, Cetinje, Narodni muzej Crne Gore, 2013.

${ }^{11}$ P. Kasandrić, Pietro II Petrović - Njegoš. La traslazione delle sue ceneri sul Lovćen, «Europa orientale», V/ XI, 1925, pp. 750-772.

12 N. Tommaseo, Dell'animo e dell'ingegno di Antonio Marinovich, Venezia, Gondoliere, 1840.

${ }^{13}$ G. Contini, Progetto per un ritratto di Niccolò Tommaseo, in ID., Altri esercizi (1942-1971), Torino, Einaudi, 1972, pp. 5-24. 
gli slavi, morlacchi, o serbi e croati, non fossero «mangiatori di sego». ${ }^{14}$ In quell'atmosfera storico-culturale Kasandrić spicca come studioso onesto e ottimo traduttore, e soprattutto come vero e proprio mediatore culturale.

È significativo che Kasandrić non sia stato solo un attivo partecipante alla vita giornalistica dalmata, ma che abbia preso anche il ruolo dello storico di questa produzione scritta con il suo studio Il giornalismo dalmato dal 1848-1860. Appunti, pubblicato nel 1899 a Zara. ${ }^{15}$ In un'epoca in cui lo stile giornalistico coincideva con la forma del saggio, Kasandrić spicca come uno degli esponenti più validi di questo genere. La breve ma ricca inchiesta sul giornalismo dalmata non vide altre edizioni, sebbene Kasandrić volesse tornare a lavorarci, come si legge in una lettera all'amico Ante Petravić:

Quel mio libricino «Il giornalismo dalmato», stampato già nel 1899 a Zara, lo troverà con difficoltà e io non ho che una copia. Dopo la guerra, l'hanno usato a fini polemici sia i nostri che gli italiani. Ho raccomandato ad un amico a Zara di procurarmi un esemplare se lo dovesse trovare da qualche parte; ma temo che non lo troverà. Pensavo di farne una nuova edizione, corretta e ampliata. Ma di mezzo ci sono vari ostacoli. ${ }^{16}$

Scritto in una prosa elegante e colta, ma nello stesso tempo dal ritmo spigliato, il trattato di Kasandrić offre un quadro dettagliato di un periodo davvero unico nella storia della regione dalmata:

Però i giornali nuovi, sorti a quelle prime aure di libertà, furono pochi ed ebbero corta vita: fuochi di paglia che divamparono troppo rapidamente e presto si spensero; mentre i vecchi avvezzi al regime della censura preventiva e vissuti fino allora in un tranquillo dormiveglia, scossi all'improvviso e galvanizzati dalle nuove idee, si agitarono concitatamente in una fittizia esuberanza di vitalità, che ne esaurì presto le forze e ne affrettò la fine. [...] Breve vita, ma rigogliosa, ma spontaneamente e lietamente affaccendata nella ricerca d'un migliore avvenire per la patria. ${ }^{17}$

In questo passo iniziale che riassume la vita giornalistica dalmata in seguito alla «patente del marzo 1848, con cui Ferdinando I largiva ai popoli dell'Austria la costituzione e la libertà di stampa», ${ }^{18}$ si possono osservare alcune peculiarità dello stile del Kasandrić saggista: periodi lunghi che si sviluppano in crescendo attraverso una serie di parallelismi elaborati sia su analogie che su contrapposizioni, dittologie, forme auliche scelte con cura.

\footnotetext{
${ }^{14}$ N. STIPČEvić, Poređenja, cit., p. 93.

${ }^{15}$ P. KASANDrić, Il giornalismo dalmato dal 1848-1860 appunti, Zara, S. Artale, 1899.

${ }^{16}$ H. Morović, Iz prepiske Petra Kasandrića sa A. Petravićem, cit., p. 529.

${ }^{17}$ P. KASANDRIĆ, Il giornalismo dalmato, cit., pp. 1-2.

${ }^{18}$ Ivi, p. 2.
} 
La struttura binaria sembra essere quella privilegiata da Kasandrić nella prosa saggistica, mentre in poesia, come si è visto, preferiva il ritmo ternario, «costrutto certamente non inventato da Kasandrić, ma presente in quasi ognuna delle sue liriche con varia funzione». ${ }^{19}$ Tuttavia la struttura a tre non è del tutto assente. Vi si notano serie di verbi («loda, incoraggia e raccomanda caldamente») o di sintagmi nominali retti dalla stessa preposizione di volta in volta ripetuta («contro le nuove disposizioni in materia di stampa, contro le tendenze reazionarie del ministero, contro il sistema d'istruzione negli istituti medi»), o dalla stessa congiunzione («e con maggiore larghezza di vedute e serietà di patriottici propositi ed elevatezza di artistici intendimenti»). Dall'altro lato il testo pullula di dittologie di varia natura, soprattutto quelle aggettivali basate sul rapporto di sinonimia: «articoli piuttosto dottrinali e cattedratici», «giovinezza randagia e avventurosa», «linguaggio sdegnoso ed acre», «bizzosa e bisbetica famiglia dei suoi collaboratori», «serio ed austero», ecc. Le strutture binarie si notano anche nei sintagmi nominali utilizzati per descrivere varie personalità della vita culturale dalmata dell'epoca: «persone di molta cultura e di indiscusso valore», «letterato raguseo di vivo e versatile ingegno e di varia cultura», "splendore di linguaggio e vigore di argomenti», «brillante ingegno e geniale poeta», ecc. Le descrizioni stesse condensano sinteticamente informazioni biografiche essenziali sugli scrittori e giornalisti dalmati, seguendo una struttura rigida e dal punto di vista sintattico molto uniforme, cui corrisponde una ricca variazione sul piano lessicale:

Mattia Ban, letterato raguseo di vivo e versatile ingegno e di varia cultura; autore in gioventù di versi romantici e di scritti politici, poeta più in questi che in quelli; scrittore di opuscoli sull'arte della guerra e sull'educazione femminile; poi tragico di bella fama e tra i più fecondi del teatro serbo e croato. [...]

Ladislao Vežić, notaio a Varasdino, dalmata di origine, nativo da Castel Venier di Novegradi, brillante ingegno e geniale poeta. [...]

Giovanni Duplančić, ultimo rampollo di agiata famiglia zaratina, culto e promettente ingegno, di spiriti liberali, serio ed austero oltre l'età, il quale aveva già fatto le sue prime armi nella Dalmazia Costituzionale. ${ }^{20}$

Kasandrić adopera un procedimento simile quando descrive la vita dei giornali di cui tratta, come nel caso della «Gazzetta di Zara»: «Animato di sentimenti patriottici e generosi, ma senza un programma netto e preciso, pencolante fra opposti principi, in lotta col Governo e più con sè

\footnotetext{
${ }^{19}$ M. Festini, Le prime liriche e ultime di Petar Kasandrić, cit., p. 262.

${ }^{20}$ P. KASANDRIĆ, Il giornalismo dalmato, cit., pp. 12, 14, 86.
} 
stesso, fu specchio fedele, se pur non molto nitido, di tutte le incertezze, di tutti i tentennamenti, di tutte le incoerenze di quell'epoca agitata». ${ }^{21} \mathrm{Il}$ soggetto è sottinteso e la proposizione principale è ritardata da una serie di anteposizioni, tipiche dello stile giornalistico. Di nuovo il parallelismo risulta la figura di stile privilegiata da Kasandrić e serve a sottolineare la tensione «di quell'epoca agitata». L'aspetto formale rispecchia quello contenutistico attraverso corrispondenze semantiche (scelta delle stesse parole «di tutte/i» o delle parole col significato uguale o simile «incertezze, tentennamenti, incoerenze») e sintattiche (lo stesso ordine di parole riportato con insistenza). L'emblematica tensione della frase di Kasandrić si sviluppa quindi su un rapporto stretto tra analogie e contrapposizioni e attraverso una sintassi poco lineare.

Oltre alle strutture sintattiche binarie o ternarie, basate su similitudine o antitesi, da queste righe emerge anche l'ironia di Kasandrić che si risolve principalmente negli accostamenti inattesi di concetti, come nell'esempio «in lotta col Governo e più con sé stesso», dove il secondo elemento crea un effetto di sorpresa. Non di rado Kasandrić fa uso di poche parole o poche righe piene di ironia accentuata dal secondo termine o elemento del sintagma che si presenta inatteso, come per esempio in un articolo che definisce «gonfio di retorica e deliziosamente ingenuo» oppure «sonetto bruttino, ma con chiusa abbastanza felice». Con altrettanta arguzia licenzia una canzonetta metastasiana «La Guardia Nazionale alle donzelle», pubblicata anonimamente sulla rivista «Le Rimembranze della settimana». Dopo aver definito una delle strofe «un massacro di metafore», conclude la stroncatura riferendosi all'anonimità dell'autore: «Chi fosse l'arcadico Tirteo della Guardia Nazionale ragusea non ci venne dato di scoprire, ma non c'è proprio da desolarsene». ${ }^{22}$ L'efficacia della subordinata che chiude il periodo ricorda quella di un Witz o di oneliner della commedia anglossassone. L'assenza di correttezza politica, che oggi (ma non nell'epoca in cui scriveva Kasandrić) sembrerebbe del tutto inappropriata in un testo simile, rende la prosa colta di Kasandrić vivace e a tratti comica.

Nella sfera del lessico emerge la propensione alle parole auliche, che Kasandrić aveva difeso in un articolo pubblicato sull' $" I$ Iskra ${ }^{23}$ in risposta alla recensione della sua traduzione dei canti epici serbi. Nella sua recensione ${ }^{24}$ piuttosto positiva Stjepan Buzolić avanzava tuttavia qualche

\footnotetext{
${ }^{21}$ Ivi, p. 96.

${ }^{22}$ Ivi, p. 22.

${ }^{23}$ P. Kasandrić, Pro bono sua, «Iskra», 1/5, 1884, pp. 19-20.

${ }^{24}$ S. Buzolić, Narodne pjesme. Canti popolari epici serbi versione metrica di Petro Cassandrić, Zara, S. Artale tipografo-editore, 1884, «Iskra», 1/4, 1884, p. 16.
} 
critica a Kasandrić, rimproverandogli di non aver incluso anche i canti lirici nella sua raccolta e sostenendo che alcuni lemmi aulici si potessero sostituire con forme più popolari. Alla prima critica Kasandrić risponde con un'analogia dicendo che un uomo, il quale avesse chiesto un parere sulle proprie rape e si sentisse dare, in risposta, opinioni su patate o altri ortaggi, potrebbe ben dire: «Ma, io le avevo chiesto cosa pensasse delle rape».$^{25}$ Con lo stesso tono canzonatorio e con molta consapevolezza delle proprie scelte Kasandrić risponde anche alla seconda critica: «Per quale motivo io in alcuni casi ho usato queste e non altre parole, più comuni e più semplici, questo credo non necessiti di spiegazioni. Chi sa cosa è la poesia, comprenderà da solo, altrimenti cancelli dalle poesie di Carducci le parole marsi militi e metta marsi soldati, cancelli alti fastigi e metta alti tetti, che tolga la parola verziere e che metta orto» ${ }^{26}$

È la dimostrazione che per Kasandrić la poesia scritta in lingua italiana, anche se si trattava di poesia tradotta, doveva necessariamente usare un codice linguistico poetico, altrimenti non era poesia. Non sorprende perciò la presenza del lessico aulico in una prosa di registro alto. Inoltre, alcune scelte lessicali ammiccano allo stile delle polemiche, di frequente in riferimento ai dibattiti accesi nella Dalmazia dell'epoca i cui argomenti spaziavano dalla nazionalità slava contrapposta a quella italiana alle riforme ortografiche: «la prurigine delle polemiche linguistiche», «stuzzicare le bizze letterarie», «gravi beghe», «trionfando delle cabale», «barbassori dell'ortografia dalmata», «batteva in breccia l'idea italiana», «metteva in burletta i retrogradi». Altre scelte quali conchiudeva per concludeva, cangiare per cambiare, omai per ormai, riesciva per riusciva non meritano particolari rilievi stilistici, essendo opzioni tipiche dell'italiano scritto dell'epoca, come ha dimostrato Luca Serianni. ${ }^{27}$

Come ho cercato di illustrare, Kasandrić domina con disinvoltura lo stile colto anche nei testi di prosa, conferendogli vivacità attraverso un'ironia spesso pungente e un tono passionale da cui traspare il suo temperamento. Dallo stesso stile prosastico, frutto della sua doppia coscienza linguistica e letteraria, emergono anche alcune idee che contrassegnano il pensiero di Kasandrić lungo tutta la sua carriera di giornalista e critico letterario. Ad esempio quella della fratellanza dei popoli slavi, che mostra la continuità del suo pensiero con quello di Tommaseo, l'autore seguito anche nel

${ }^{25}$ P. KasAndrić, Pro bono sua, cit., p. 19.

${ }^{26}$ Ivi, p. 20.

${ }^{27}$ L. Serianni, Lingua poetica italiana. Grammatica e testi, Roma, Carocci editore, 2009, in particolare pp. 186-193. 
metodo traduttivo applicato ai canti popolari serbi e croati. ${ }^{28}$ La simpatia per l'unione degli slavi del Sud a volte prende respiro più ampio, come dimostra l'esempio seguente:

Trovandosi ancora a Vienna le deputazioni nazionali boema e polacca, e parecchi Serbi e Slovacchi della Monarchia, si tenne quello stesso giorno la prima adunanza degli Slavi dell'Austria, a cui prese parte anche la deputazione croata; in quell'incontro apparve la prima volta - splendido miraggio - la solidarietà dei popoli Slavi della Monarchia. ${ }^{29}$

Ragionamenti simili si hanno in una lunga nota in cui Kasandrić voleva giustificare l'impiego di certa terminologia in riferimento alla lingua parlata dai serbi e dai croati in Dalmazia nel periodo di cui trattava:

I termini "lingua illirica" e "lingua slava", sebbene antiquati ed impropri, vengono adoperati in tutto questo lavoro, perché trovandosi nelle citazioni che vi ricorrono, l'usare quello di "lingua croata" avrebbe in alcuni casi determinato, coi criteri d'oggi, contraddizioni ed equivoci. - Al tempo di cui trattassi in questi cenni, i parlanti italiano dicevano illirica o più ordinariamente slava la lingua dei Croati e dei Serbi, che, comune ad entrambi, viene chiamata croata dai primi, serba dai secondi. Allora, in Dalmazia, all'udirla chiamare così o colà nè gli uni nè gli altri si formalizzavano; oggi in certi casi, ad usare una locuzione invece dell'altra si commette uno sproposito $\mathrm{e}$ - secondo l'intenzione che ci si mette o vi si attribuisce - perfino un'ingiuria. - Per ciò abbiamo preferito lasciare la vecchia dizione; la quale ha pure il vantaggio di esprimere meglio quella concordia di sentimenti e di intendimenti che allora affratellava le due stirpi. ${ }^{30}$

La stessa idea della concordia e fratellanza tra le due stirpi viene espressa nel già citato articolo dedicato a Tommaseo: «Tommaseo, che si sacrificò molto per il popolo italiano, abbracciava con lo stesso amore tutte le stirpi del nostro popolo». ${ }^{31}$ Allo stesso periodo risale una considerazione espressa nella lettera ad Ante Petravić e rivolta al mondo letterario tripartito,

${ }^{28}$ Kasandrić sembra seguire il metodo conciliante di Tommaseo elaborando una traduzione che è sia addomesticante (il ricorso alla lingua poetica italiana) che estraniante (l'uso del decasillabo). Su un piano più specifico, l'influenza di Tommaseo si nota in alcune scelte lessicali particolari, come dimostrano alcuni versi del Marko Kraljević i Musa Kesedžija nelle due traduzioni: Sgraziato, non t'ho io detto / Di non fare in domenica zuffa? / Vergogna è, due contr'uno (Tommaseo) e Non t'ho detto, misero, che mai / Non attacchi zuffa la domenica? / Due contr'uno gli è vergogna, Marco! (Kasandrić).

${ }^{29}$ P. KASANDRIć, Il giornalismo dalmato, cit., p. 39, corsivo mio.

${ }^{30}$ P. KASANDrić, Il giornalismo dalmato, cit., p. 90, corsivo mio.

${ }^{31}$ P. Kasandrić, Iz Tomazeove prepiske od 1839. do 1849, «Novi Život», XVIII/5, 1924, pp. 129-135: 135. 
ma unito nella visione di Kasandrić: «Io vorrei che tutti e tre i rami del nostro albero letterario si sviluppassero con altrettanta ricchezza: a Belgrado, a Sarajevo, a Zagabria, e che ciascuno preservasse la propria peculiarità». ${ }^{32}$ Sulla stessa scia prosegue anche l'articolo dedicato a Njegoš, di cui dice: «A Roma non faceva visite ufficiali, ma riceveva molti preti e frati cattolici dalla Croazia, dalla Slavonia e dalla Dalmazia ai quali parlava con calore dell'unità della patria, a cui bisogna tendere senza preoccupazione nè pregiudizio delle differenti credenze religiose, perché la fede non vieta a nessuno di amare la propria lingua e la propria nazione». ${ }^{33}$

Kasandrić coltiva con coerenza l'illirismo (e in seguito lo jugoslavismo) ${ }^{34}$ durante un lungo periodo di cambiamenti storici, sia nella corrispondenza privata che nelle opere stampate. Sebbene le sue opinioni siano visibili nel trattato sul giornalismo (e altrove) - è chiaro, ad esempio, che sarebbe stato favorevole alla riforma ortografica promossa da Ljudevit Gaj -, si ha l'impressione che le prese di posizione personali non influiscano sul suo ragionamento da critico: «Proprio allora battagliavano fra di loro gli scrittori dalmati e croati intorno alla nuova ortografia del Gaj; i linguaioli da una parte e dall'altra disputavano di purismo e di eloquio popolare, qua con vivacità un po' caustica, là con burbanza pedantesca, spropositando un po' tutti». ${ }^{35} \mathrm{Nel}$ suo stile elegante, dalle chiuse efficaci, Kasandrić non risparmia nessuno.

Petar Kasandrić merita indubbiamente una maggiore attenzione da parte degli studiosi. Spero di avere l'occasione di tornare a occuparmene in maniera più vasta e profonda. Questo studio è un primo avvicinamento a Kasandrić, offerto in omaggio a chi con lui condivide un'intima conoscenza della versificazione italiana e l'idea della fratellanza dei popoli slavomeridionali, espressa e sostenuta anche in tempi non facili.

${ }^{32}$ H. Morović, Iz prepiske Petra Kasandrića sa A. Petravićem, cit., p. 528.

${ }^{33}$ P. Kasandrić, Pietro II Petrović - Njegoš, cit., p. 770.

${ }^{34}$ In riferimento a varie espressioni di illirismo, si veda E. Ivetić, Jugoslavia sognata. Jugoslavismo dalle origini, Milano, FrancoAngeli, 2012.

${ }^{35}$ P. KasAndrić, Il giornalismo dalmato, cit., p. 6. 


\title{
Marija Bradaš
}

\section{O ESEJISTIČKOJ PROZI PETRA KASANDRIĆA}

\author{
(Rezime)
}

Predstavljajući razne vidove bavljenja uspostavljanjem veza između dve jadranske obale Petra Kasandrića, od prevodilačkog do kritičko-žurnalističkog, autorka se u radu bavi i analizom osobenosti esejističke proze ovog dalmatinskog kulturnog poslenika na italijanskom, ukazujući da se vešto služi, kao neko ko je jednako usvojio oba jezička koda, i onaj srpsko-hrvatski i italijanski, leksičkim, stilskim i retoričkim postupcima tipičnim za određene forme pisanog izraza na italijanskom. 